\title{
Correlation between Structures and Antioxidant Activities of Polyvinylpyrrolidone/Garcinia mangostana L. Extract Composite Nanofiber Mats Prepared Using Electrospinning
}

\author{
Ida Sriyanti, ${ }^{1,2}$ Dhewa Edikresnha, ${ }^{1,2}$ Annisa Rahma, ${ }^{3}$ Muhammad Miftahul Munir, ${ }^{1,2}$ \\ Heni Rachmawati, ${ }^{3,4}$ and Khairurrijal Khairurrijal ${ }^{1,2}$ \\ ${ }^{1}$ Department of Physics, Faculty of Mathematics and Natural Sciences, Institut Teknologi Bandung, Bandung 40132, Indonesia \\ ${ }^{2}$ Bioscience and Biotechnology Research Center, Institute for Research and Community Services, Institut Teknologi Bandung, \\ Bandung 40132, Indonesia \\ ${ }^{3}$ Pharmaceutics Research Division, School of Pharmacy, Institut Teknologi Bandung, Bandung 40132, Indonesia \\ ${ }^{4}$ Nanoscience and Nanotechnology Research Center, Institute for Research and Community Services, Institut Teknologi Bandung, \\ Bandung 40132, Indonesia
}

Correspondence should be addressed to Khairurrijal Khairurrijal; krijal@fi.itb.ac.id

Received 31 March 2017; Accepted 1 June 2017; Published 18 July 2017

Academic Editor: Silvia Licoccia

Copyright (C) 2017 Ida Sriyanti et al. This is an open access article distributed under the Creative Commons Attribution License, which permits unrestricted use, distribution, and reproduction in any medium, provided the original work is properly cited.

Nanofiber mats of polyvinyl(pyrrolidone) (PVP) with Garcinia mangostana extract (GME) as the encapsulated drug have been developed using electrospinning. SEM images of all electrospun PVP/GME composite nanofiber mats showed that they had similar and smooth morphology, no beads, and spindle shape. Its average diameter decreased and its surface area therefore increased with the decrease of its PVP concentration. The benefit of high surface area is obvious in drug delivery systems for poorly water-soluble drugs. Their FTIR spectra indicated that PVP and GME interacted intermolecularly via hydrogen bonds in the composite nanofiber mats. A conformational change in the $\mathrm{C}-\mathrm{H}$ chain of PVP occurred in the composite nanofiber mats due to the intermolecular interactions. Their XRD patterns confirmed that they were amorphous because of amorphization during electrospinning. The XRD analyses also strengthened the FTIR studies; namely, GME and PVP formed intermolecular interactions in the electrospun composite nanofiber mats. As a result, GME as the encapsulated drug was molecularly dispersed in the electrospun PVP nanofiber matrix that functioned as a drug delivery system. From the 2,2-diphenyl-1-picrylhydrazyl (DPPH) assay, the composite nanofiber mats exhibited very high antioxidant activities despite having been exposed to high voltage during electrospinning. Therefore, they are potential antioxidant products for food and pharmaceutics.

\section{Introduction}

Mangosteen (Garcinia mangostana L.) is widely cultivated across Indonesia. The color of mangosteen is dark purple or reddish with white, soft, and juicy edible pulp. It has slightly sour but sweet flavor as well as pleasant aroma. The fruit has been used as traditional medicine to treat abdominal pain, diarrhea, dysentery, infected wound, suppuration, and chronic ulcer. The major active substances in mangosteen pericarp are xanthones and their derivatives such as $\alpha-, \beta-, \gamma-$ mangostin, garcinone, mangostanol, and gartinin, in which $\alpha$-mangostin is the major xanthone [1]. They are classified into polyphenolic compounds and have been reported to have high antioxidant activities [2-4]. Some delivery methods to improve the bioavailability of $\alpha$-mangostin have recently been studied, including microgel [5], solid dispersion [6], microsphere [7], and nanofiber mat [8].

Electrospinning is a method to produce nanofiber mats with a very large surface area to volume ratio from a precursor polymer solution. An electrostatic field is applied to the precursor polymer solution to induce positive charges on the surface of the polymer solution so that when it is subjected to a very high potential difference, the positively charged polymer solution will be attracted towards the grounded 
collector to form nanometer-size fibers $[9,10]$. By adjusting properties of the polymer solution and electrostatic field during the electrospinning process, the morphology and size of the resulting fibers can be controlled. Recently, it has been reported that highly uniform polymer nanofibers could be obtained by maintaining a constant current [11]. Although the simple and straightforward electrospinning processes have developed very quickly to prepare a wide variety of complicated nanostructures such as core-sheath, Janus, and tri-layer [12-14], the mainstream is still the monolithic nanocomposites resulted from the single-fluid electrospinning processes, in which an active ingredient is homogeneously distributed within the polymeric matrix to take advantages of the nanofibers huge surface area.

Polyvinylpyrrolidone (PVP) along with polyvinyl alcohol (PVA) and polyethylene glycol (PEG) are vinyl polymers that are widely used to develop products of drug delivery media [15]. Moreover, PVP is a widely used hydrophilic polymer because it is nontoxic, electrospinnable, and soluble in water, and it also has good biocompatibility [16-19]. It has also been reported that electrospun PVP nanofibers had an effective release control and it was able to increase the solubility of curcumin [20]. Although many studies have been done to load GME into polymer nanofibers, the use of PVP nanofibers, however, is rarely explored. Very recently, a short description of electrospun PVP/GME composite nanofiber mats along with the release of GME from the nanofibers has been given [18]. In this paper, we will report their antioxidant activities investigated by using the 1.1diphenyl-2-picrylhydrazyl (DPPH) assay. Their structures were also analyzed by employing a scanning electron microscope (SEM), an X-ray diffractometer (XRD), and a Fourier transforms infrared (FTIR) spectrometer. The relationship between their structures and antioxidant activities will be thoroughly discussed.

\section{Materials and Methods}

2.1. Materials. Polyvinylpyrrolidone (PVP) (MW $1,300 \mathrm{~kg} \cdot \mathrm{mol}^{-1}$ ) and 2,2-diphenyl-1-picrylhydrazyl (DPPH) and $\alpha$-mangostin standard were purchased from Sigma-Aldrich, Indonesia. Mangosteen pericarps were obtained from a local market in Bandung, Indonesia. Technical grade ethanol 96\% for maceration was acquired from Bratachem, Indonesia, while analytical grade methanol used for antioxidant tests was purchased from Merck, Indonesia.

2.2. $\alpha$-Mangostin Content Assay. GME paste was obtained from mangosteen pericarp extraction using the maceration method. The $\alpha$-mangostin content in the GME paste and nanofiber mats was analyzed using High Performance Liquid Chromatography (HPLC). The sample was injected into a C-18 column (Phenomenex, $250 \mathrm{~mm} \times 4,6 \mathrm{~mm}$, particle size $5 \mu \mathrm{m})$ using a HPLC system with a UV spectrophotometer detector (Shimadzu, SPD-20A/LC-20AD). The mobile phase was water-methanol $(95: 5)$ with flow rate of $1 \mathrm{~mL} / \mathrm{min}$. The GME was detected at wavelength of $320 \mathrm{~nm}$.
TABLE 1: Precursor mixture solution for electrospinning process.

\begin{tabular}{lcc}
\hline Precursor mixture solution & PVP (wt.\%) & GME (gram) \\
\hline PM5 & 5 & 0.3 \\
PM7 & 7 & 0.3 \\
PM10 & 10 & 0.3 \\
\hline
\end{tabular}

2.3. Preparation of Polymer Mixture. PVP was dissolved in ethanol to obtain PVP solutions with the concentrations of 5,7 , and $10 \%$ (wt.\%) with the total mass of each solution being 20 grams. Each solution was mixed using a magnetic stirrer for about one hour at $40^{\circ} \mathrm{C}$, followed by the addition of GME paste as much as 0.3 gram into the solution. Each mixture was mixed further using a magnetic stirrer for about 2 hours at $40^{\circ} \mathrm{C}$ to make a precursor mixture solution. A mixture of the PVP solution and the GME paste was stirred at room temperature for $30 \mathrm{~min}$ until a clear and homogeneous solution was once again achieved. The precursor mixture solution is given in Table 1 .

2.4. Electrospinning Process. The electrospinning apparatus (Nachriebe 600) schematically described in Figure 1 was employed to produce nanofiber mats. Ten $\mathrm{mL}$ of the precursor solution was inserted into a syringe with the needle diameter of $0.45 \mathrm{~mm}$, then electrospun, and collected at a grounded drum collector that was placed at a distance of $10 \mathrm{~cm}$ from the tip of the needle. The solution flow rate, the applied voltage, the humidity, and the time for electrospinning were $0.5 \mathrm{~mL} / \mathrm{h}, 15 \mathrm{kV}, 40-50 \%$, and about 10 h., respectively. The PVP/GME composite nanofiber mats that were obtained from the PM5, PM7, and PM10 precursors were called the PM5, PM7, and PM10 composite nanofiber mats, respectively.

2.5. Scanning Electron Microscopy. Morphologies of the PM5, PM7, and PM10 composite nanofiber mats were analyzed by a scanning electron microscope (SEM) (JEOL, JSM-6510). Samples were coated with electric conductive carbon. The SEM images were observed with $10 \mathrm{kV}$-voltage and 10,000time optical magnification. The fibers size distribution was analyzed using the Origin ver. 8 software.

2.6. Fourier-Transform Infrared Spectroscopy. Molecular interactions between PVP and GME in the PM5, PM7, and PM10 composite nanofiber mats were evaluated using a Fourier-transform infrared (FTIR) spectrometer (Bruker, Alpha). The FTIR spectra were recorded with the wavenumber in the range of 500 to $4000 \mathrm{~cm}^{-1}$.

2.7. X-Ray Diffraction Pattern. X-ray diffraction patterns of GME, PVP, and the PM5, PM7, and PM10 composite nanofiber mats were recorded using an X-ray diffractometer (XRD) (Rigaku, SmartLab) under the following conditions: a $\mathrm{Cu}$ target tube as a source of radiation, $\mathrm{K}_{\alpha}$ and $\mathrm{K}_{\beta}$ lines, applied voltage of $40 \mathrm{kV}$, and current of $35 \mathrm{~mA}$. A sample was placed on a sample holder and irradiated with an X-ray beam. 


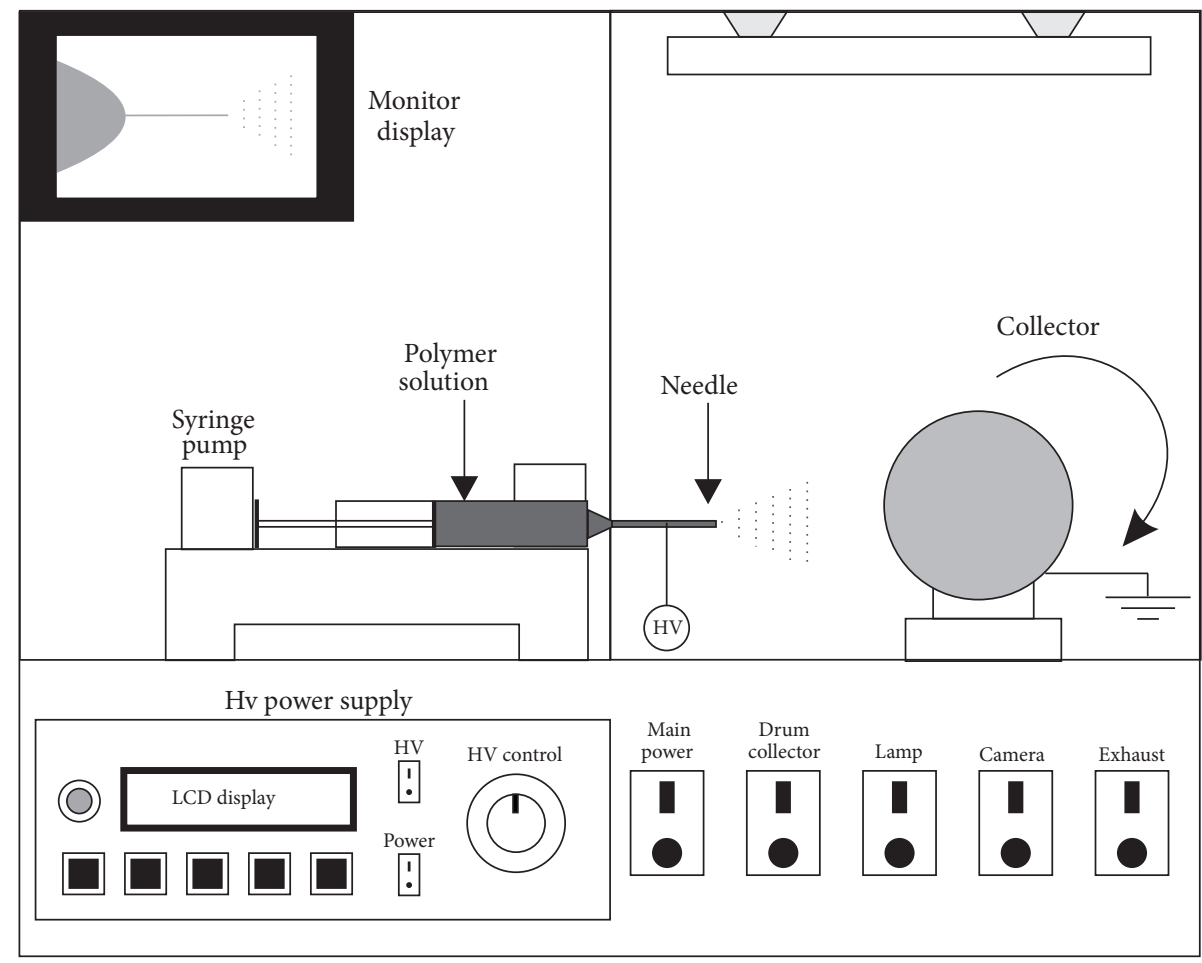

FIgURE 1: The schematic diagram of electrospinning apparatus used in the experiments.

The diffraction patterns were recorded with the $2 \theta$ position in the range of 5 to $70^{\circ}$.

2.8. Antioxidant Activity. In vitro test of antioxidant activity was performed to study whether the electrospinning process with its high voltage affected the biological activity of GME. The antioxidant activity was tested on the GME, PM5, PM7, and PM10 composite nanofiber mats using the DPPH assay. Each sample was made by dissolving the mat in analytical methanol at different concentrations. One $\mathrm{mL}$ of the sample solution was then mixed with $1 \mathrm{~mL}$ of $50 \mu \mathrm{g} / \mathrm{mL} \mathrm{DPPH}$ (thus, at a volume ratio of $1: 1$ ) and allowed to stand for $30 \mathrm{~min}$. The change of absorbance of DPPH was measured using a UV-Vis spectrophotometer (Beckman, DU 7500i) at the wavelength of $515 \mathrm{~nm}$. Methanol was used as the blank solution, a 50$\mu \mathrm{g} / \mathrm{mL}$ DPPH solution as the control, and ascorbic acid as the equivalent standard or the positive control of the antioxidant test. The antioxidant activity (AA) is expressed as [21]

$$
\% \mathrm{AA}=\frac{\left(A_{\text {control }}-A_{\text {sample }}\right)}{A_{\text {control }}} \times 100,
$$

where $A_{\text {control }}$ is the absorbance of the DPPH solution and $A_{\text {sample }}$ is the absorbance of the sample (a nanofiber mat). Further, a curve that represents the relationship between the concentration of $\alpha$-mangostin and $\%$ AA is plotted with the concentration of $\alpha$-mangostin as the $x$-axis and \% AA as the $y$-axis. A regression line is then fitted to obtain an equation $(y=m x+c)$. The $\alpha$-mangostin concentration that is expected to exhibit $50 \%$ inhibition ( $\mathrm{IC}_{50}$ value) is determined by finding the $x$-value resulting in the $y$-value of $50 \%$. The amount of $\alpha$-mangostin in GME and nanofibers of PM5, PM7, and PM10 have to be the same. The amount of $\alpha$-mangostin was determined by HPLC (Agilent Technology, USA).

\section{Results and Discussion}

3.1. $\alpha$-Mangostin Content Assay in the GME and Nanofibers Mat. The group of compounds in GME which plays a role in a wide range of bioactivity is $\alpha$-mangostin [5]. To measure the level of $\alpha$-mangostin in GME and nanofiber mats, a quantitative analysis had been conducted using HPLC (Agilent Technology, USA). The standard curve of $\alpha$ mangostin $(\mathrm{mg} / \mathrm{mL})$ is shown in Figure 2 . The result showed that the amount of $\alpha$-mangostin in the GME was $13 \%$ and the nanofiber mats of PM5, PM7, and PM10 were 9.8\%, 8.1\%, and $6.5 \%$, respectively.

3.2. SEM Images. Figures 3(a)-3(c) show the morphology of PVP nanofiber mats without GME (5, 7, and $10 \mathrm{wt} . \%)$ obtained using SEM with a magnification of 10,000 times. The SEM images showed that the PVP (5 wt.\%) produced fibers with beads. Along with the increasing concentration of polymer PVP to be $7 \mathrm{wt} . \%$ and $10 \mathrm{wt}$.\%, beaded-free fibers were obtained. These findings are closely correlated to the viscoelastic jet break-up theory. It has been established that the formation of beads is associated with viscoelasticity of the solution [22]. In electrospinning process, viscoelastic force allows continuous and smooth elongation of the jet into fibers [23]. The elongation involves fiber thinning as it travels to the collector. In a low viscosity solution, polymer 


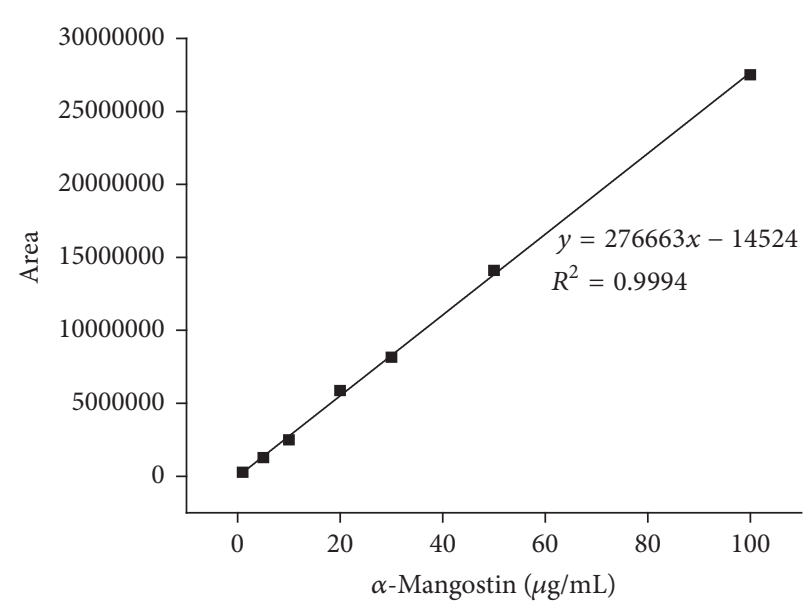

FIGURE 2: Standard curve of $\alpha$-mangostin $(\mu \mathrm{g} / \mathrm{mL})$.

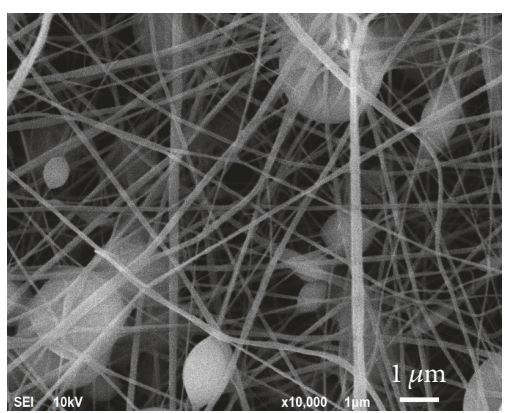

(a)

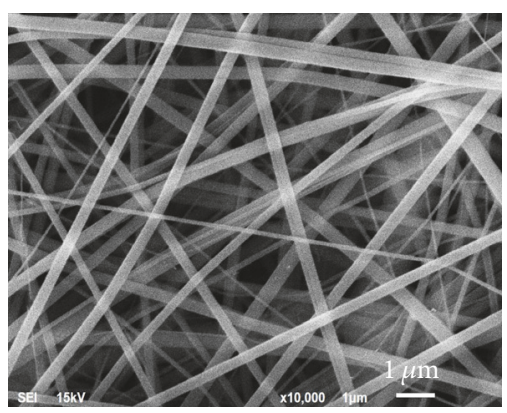

(d)

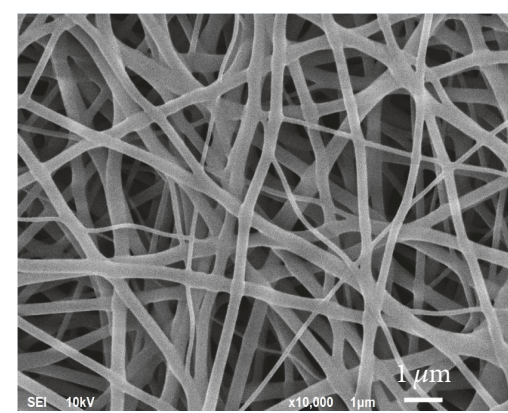

(b)

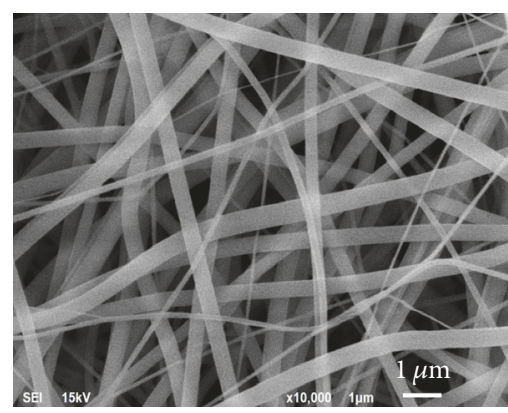

(e)

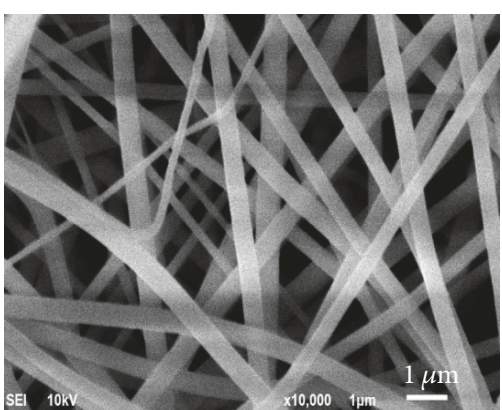

(c)

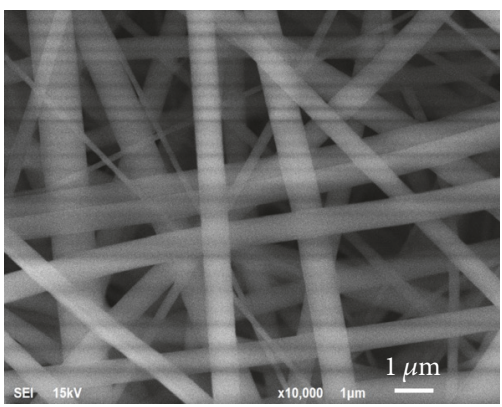

(f)

FIGURE 3: SEM images (10,000x) of PVP nanofiber mats (without GME) with concentrations (a) 5 (wt.\%), (b) 7 (wt.\%), (c) 10 (wt.\%) and (d) PM5, (e) PM7, (f) PM10 composite nanofiber mats.

chain entanglement is limited and the fiber thinning process eventually results in fibers break-up and formation of beads [9]. At this point, the polymer chains have fully been extended and the inertial force of beaded form is more dominant than the viscous force, which causes resistance to form fiber [24]. After relaxation time of the polymer is achieved, the beads might stretch out into fiber again and these events lead to a microscopic characteristic known as beads-on-string [22]. A high viscoelastic characteristic can be achieved by putting more highly extensible compounds (polymers) into the precursor solution [22]. Consequently, as the concentration of
PVP in the solution increased, fibers without beads were successfully achieved.

The addition of GME into the solution of PVP $(5,7$, and $10 \mathrm{wt} . \%)$ increased the viscosity and lowered the surface tension in which both of these factors can affect morphology and the diameter of the nanofiber mats [9]. Table 2 illustrates the viscosity and surface tension of the solution PVP/GME. Overall, the viscosity of precursor solution of the PVP/GME composite nanofiber mats was higher than the precursor solution of PVP nanofiber mats (without GME). It was also found that a significant increase of viscosity 
TABLE 2: The viscosity and surface tension of precursor solution of PVP/GME.

\begin{tabular}{|c|c|c|c|}
\hline \multicolumn{2}{|c|}{$\begin{array}{l}\text { Composition of precursor } \\
\text { solution }\end{array}$} & \multirow[t]{2}{*}{ Viscosity (cP) } & \multirow{2}{*}{$\begin{array}{l}\text { Surface tension } \\
\quad(\text { dyne } / \mathrm{cm})\end{array}$} \\
\hline PVP (wt.\%) & GME (gram) & & \\
\hline 5 & - & 12.23 & 34.26 \\
\hline 7 & - & 25.42 & 33.03 \\
\hline 10 & - & 70.1 & 32.25 \\
\hline 5 & 0.3 & 27.48 & 32.76 \\
\hline 7 & 0.3 & 60.32 & 31.50 \\
\hline 10 & 0.3 & 98.88 & 30.84 \\
\hline
\end{tabular}

and slight decreases of surface tension resulted as GME content increased. As GME was added to the PVP solution, the viscosity of the solution increased because there were more solutes in the PVP solution and GME might promote the entanglements of PVP molecules. Therefore, from the experimental result, it can be concluded that the viscosity appears to affect the morphology of composite fiber mats more significantly.

Figures 3(c)-3(d) show the SEM images of PM5, PM7, and PM10 composite nanofiber mats. They showed similar and smooth morphology but different size and all of them had diameters in nanometer size and were perfectly beaded-free with cylindrical shape. On the other hand, at the macroscopic level, it can be clearly seen that the colors of the composite fiber mats were yellow. These microscopic findings indicated that the GME had evenly been mixed into the PVP polymer matrix.

The nanofiber size distributions of the obtained composite nanofiber mats are shown in Figures 4(a)-4(c) for the PVP nanofiber mats (without GME) and the composite nanofiber mats. For the PVP nanofiber (5 wt.\%), the diameter was distributed from 50 to $300 \mathrm{~nm}$ with the average diameter $\left(D_{\text {ave }}\right)$ of $143 \mathrm{~nm}$ and the standard deviation (SD) of $38 \mathrm{~nm}$. The range, $D_{\text {ave }}$, and SD of the PVP nanofiber (7wt.\%) were 50 to $450 \mathrm{~nm}, 248 \mathrm{~nm}$, and $65 \mathrm{~nm}$, respectively. The range, $D_{\text {ave }}$, and SD of the PVP nanofiber (10 wt.\%) were 50 to $650 \mathrm{~nm}$, $322 \mathrm{~nm}$, and $92 \mathrm{~nm}$, respectively. In Figures 4(d)-4(f), for the PM5 composite nanofiber mat, the diameter was distributed in the range of 50 to $450 \mathrm{~nm}$ with the average diameter $\left(D_{\text {ave }}\right)$ of $217 \mathrm{~nm}$ and the standard deviation (SD) of $82 \mathrm{~nm}$. The range, Dave, and $\mathrm{SD}$ of the $\mathrm{PM} 7$ composite nanofiber mat were 75 to $650 \mathrm{~nm}, 323 \mathrm{~nm}$, and $128 \mathrm{~nm}$, respectively. The range, $D_{\text {ave }}$, and SD of the PM10 composite nanofiber mat were 125 to $1200 \mathrm{~nm}, 421 \mathrm{~nm}$, and $174 \mathrm{~nm}$, respectively. Generally, the diameter of PVP/GME composite nanofiber mats was greater than the PVP nanofiber mats (without GME). It is clearly seen that the average diameter of nanofibers became larger with the increasing concentration of PVP and also as the GME was added to the PVP solution. This result is due to the fact that the increase in polymer concentration enhances the entanglement of polymer chains in the solution [25]. In addition, by increasing the concentration of polymer dan GME, the solvent will dry up faster so there will be less available time for the electrostatic coulombic force to stretch the fiber that will ultimately result in the larger diameter of fibers on the drum collector. This finding is similar to that which has very recently been reported for curcumin in PVP fibers [20].

Noting that a distribution is homogeneous when it has a ratio of the standard deviation to the average fiber diameter, which is called a coefficient of variance $(\mathrm{CV})$, less than 0.3 [26], the PM5, PM7, and PM10 composite nanofiber mats with the CVs of $0.38,0.39$, and 0.41 , respectively, were less homogeneously distributed. The lower homogeneity of fibers might be caused by the fluctuation of current during the electrospinning process [11] as the PID control action to keep a constant current was not applied to the present electrospinning system. Another factor that might contribute to the lower homogeneity was the use of high voltage of $15 \mathrm{kV}$. The high voltage can cause imbalance between precursor loading and charge-induced spinning. Consequently, one or more side jet(s) were formed to produce fibers with inconsistent sizes $[9,20]$.

3.3. FTIR Characteristics. FTIR analysis was performed to identify functional groups as a part of characterization of GME, PVP nanofiber mats, and PVP/GME composite nanofiber mats and their peaks are shown in Figure 5. One of the FTIR characteristic peaks of the GME was observed at $3273 \mathrm{~cm}^{-1}$, which was assigned for O-H stretching [5]. This indicated the presence of polyphenol in GME. The other peaks appearing at 1638, 1440,1279, and $1077 \mathrm{~cm}^{-1}$ represented $\mathrm{C}=\mathrm{O}$ stretching of carbonyl group, aromatic $\mathrm{C}=\mathrm{C}$ stretching, C-O-C stretching of methoxy group, and $\mathrm{C}-\mathrm{O}-\mathrm{C}$ stretching, respectively $[27,28]$. C-H stretching from methyl groups was indicated by two peaks at 2853 and $2923 \mathrm{~cm}^{-1}$ [5]. The GME was therefore rich in hydroxyl $(\mathrm{O}-\mathrm{H})$, carbonyl $(\mathrm{C}=\mathrm{O})$, and methoxy $(\mathrm{C}-\mathrm{O}-\mathrm{C})$ groups as previously found [28]. Similarly, the FTIR spectrum of the PVP nanofiber showed $\mathrm{O}-\mathrm{H}$ stretching at $3420 \mathrm{~cm}^{-1}$ because PVP exhibits mesomeric structures that make it possible to form hydrogen bonds between $\mathrm{C}=\mathrm{O}$ groups of pyrrolidone rings in the presence of moisture $[16,29]$. The characteristic peaks of PVP appeared at $1649 \mathrm{~cm}^{-1}$, which belongs to $\mathrm{C}=\mathrm{O}$ stretching of pyrrolidone rings, along with 1288 and $577 \mathrm{~cm}^{-1}$, which represent their $\mathrm{C}-\mathrm{N}$ stretching and in-plane $\mathrm{N}-\mathrm{C}=\mathrm{O}$ bending, respectively $[20,30]$. Asymmetric stretching of $\mathrm{CH}_{2}$ and $\mathrm{C}$ $\mathrm{H}$ deformations was indicated by the peaks at 2953 and $1423 \mathrm{~cm}^{-1}$, respectively [20, 31].

The PM10, PM7, and PM5 composite nanofiber mats had the O-H stretching peaks at 3393, 3391, and $3377 \mathrm{~cm}^{-1}$, respectively, which are higher than that of the GME at $3273 \mathrm{~cm}^{-1}$. It implied that the $\mathrm{O}-\mathrm{H}$ stretching peak of the PVP/GME composite nanofiber mat shifted to a higher wavenumber with increasing its PVP concentration. Therefore, these shifts indicated that the PM5, PM7, and PM10 composite nanofiber mats had intermolecular interactions between GME and PVP via hydrogen bonds formed between $\mathrm{OH}$ groups of GME and carbonyl groups of PVP [20, 32]. Moreover, it was also clearly seen that the shift with the highest intensity occurred for the PM5 composite nanofiber mat (see the insert in Figure 4), which had the smallest 


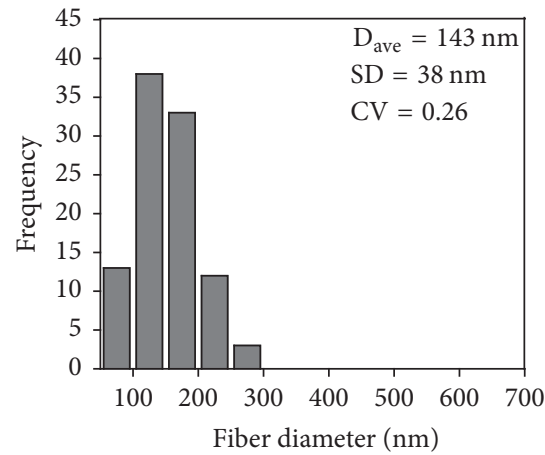

(a)

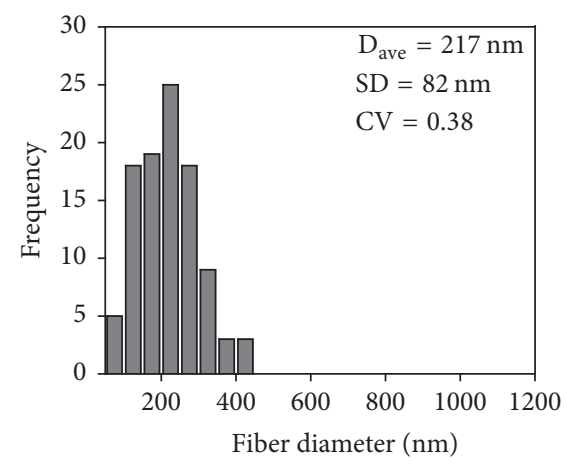

(d)

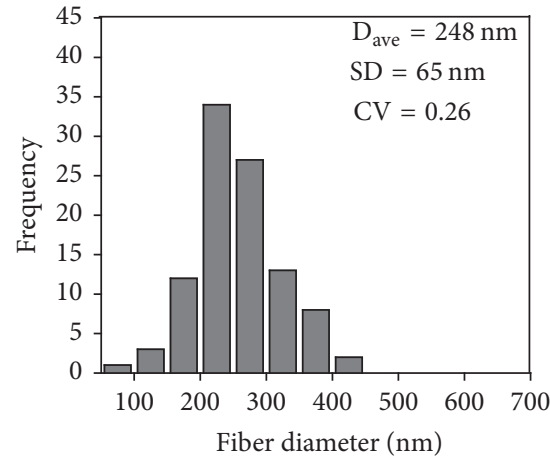

(b)

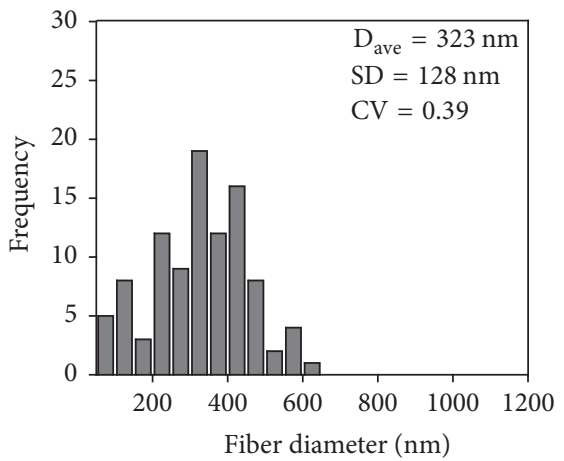

(e)

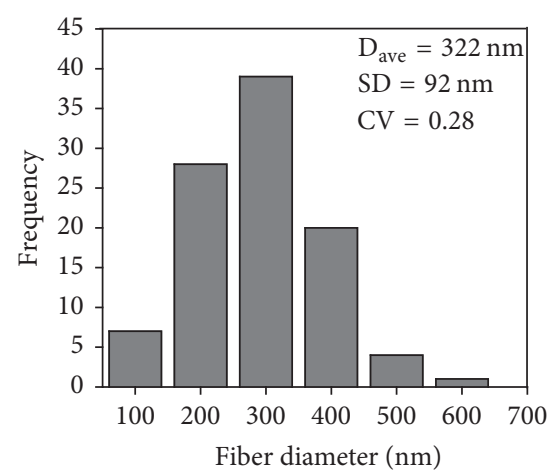

(c)

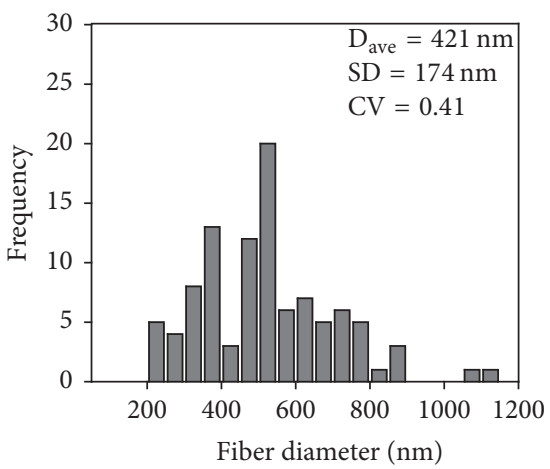

(f)

Figure 4: The diameter distributions of PVP nanofiber mats (without GME) with concentrations (a) 5 (wt.\%), (b) 7 (wt.\%), (c) 10 (wt.\%) and (d) PM5, (e) PM7, (f) PM10 composite nanofiber mats.

average fiber diameter. This was then thought as a result of the highest number of hydrogen bonds in the PM5 composite nanofiber mat that had the highest concentration of GME in the PVP nanofiber mat. The formation of hydrogen bond was also indicated by XRD analysis, which will be described in detail later. This result agrees well with our findings on HPLC study which stated that the content of $\alpha$-mangostin of GME in the PM5 composite nanofiber mat was higher than those of the PM7 and PM10 composite nanofiber mats.

The peaks at $2948 \mathrm{~cm}^{-1}, 2921 \mathrm{~cm}^{-1}$, and $2921 \mathrm{~cm}^{-1}$ were associated with the $\mathrm{CH}_{2}$ asymmetric stretching of the PM10, PM7, and PM5 composite nanofiber mats, respectively, which are lower than that of PVP powder at $2952 \mathrm{~cm}^{-1}$. It suggested that the peak of the $\mathrm{CH}_{2}$ asymmetric stretching of the PVP/GME composite nanofiber mat shifted to a lower wavenumber with increasing its GME concentration. We presumed that there was a higher conformational change in the $\mathrm{C}-\mathrm{H}$ chain with increasing the GME concentration in the PVP/GME composite nanofiber mat. However, such transition was small (the shift of $2952 \mathrm{~cm}^{-1}$ to $2948 \mathrm{~cm}^{-1}$ ) in the PM10 composite nanofiber mat, indicating that the conformational change of $\mathrm{C}-\mathrm{H}$ chain in the PM10 composite nanofiber mat that had the lowest GME concentration was insignificant. Moreover, the presence of GME in PVP nanofibers caused the peak of C-H deformation of PVP at $1421 \mathrm{~cm}^{-1}$ to shift to $1439 \mathrm{~cm}^{-1}$ for the PM5, PM7, and PM10 composite nanofiber mats. These shifts might be affected by the intermolecular interaction in the PM5, PM7, and PM10 composite nanofiber mats.

3.4. XRD Patterns. Diffraction patterns of the GME, PVP nanofiber mat, and the PM5, PM7, and PM10 composite nanofiber mats are displayed in Figure 6. Crystalline state of the GME was indicated by sharp peaks at the $2 \theta$ position of $6.12,12.12$, and $13.54^{\circ}$. This diffraction pattern is similar to that of crystalline $\alpha$-mangostin, which is the major compound of GME [33]. Meanwhile, sharp peaks with high intensity were absent in the PVP nanofiber diffraction pattern. Instead, two broad haloes appeared within the $2 \theta$ positions of 5 and $30^{\circ}$, in which the later had lower intensity and a shoulder. This diffraction pattern strongly indicated the amorphous state of PVP as formerly reported [17].

The change in crystallinity of GME in nanofibers form was found in the presence of PVP. The characteristic peaks of GME at the $2 \theta$ position of $6.12,12.12$, and $13.54^{\circ}$ were not found in the PM5, PM7, and PM10 composite nanofiber mats. This indicated the conversion of crystalline state of GME into its amorphous state. The major reason for this finding was the amorphization during the electrospinning process. When the precursor solution was ejected from the needle under an applied voltage, the accelerated positive ion movement caused the solution to undergo elongation, solvent evaporation, and liquid-to-solid transition at once [20]. As a result, PVP and GME were unable to do molecular 


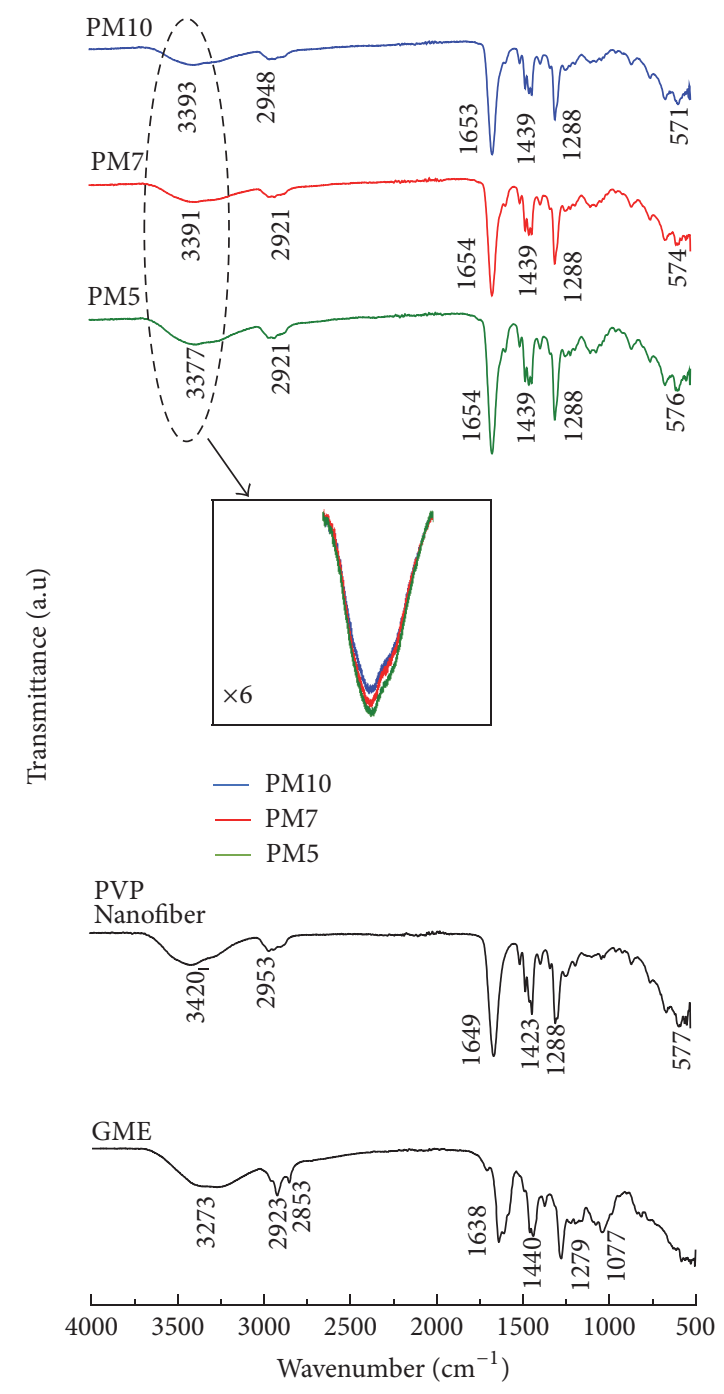

(a)<smiles>CC(C)=CCC(C(=O)O)C(O)=CCC(C)CN1CCCCC1=O</smiles>

(b)

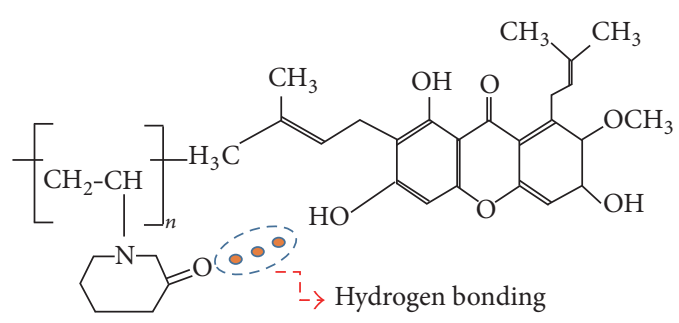

(c)

Figure 5: (a) FTIR spectra of GME, PVP nanofiber mat, and the PM5, PM7, and PM10 composite nanofiber mats; (b) molecular structures of $\alpha$-mangostin and PVP; (c) hydrogen bonding between $\alpha$-mangostin and PVP. 


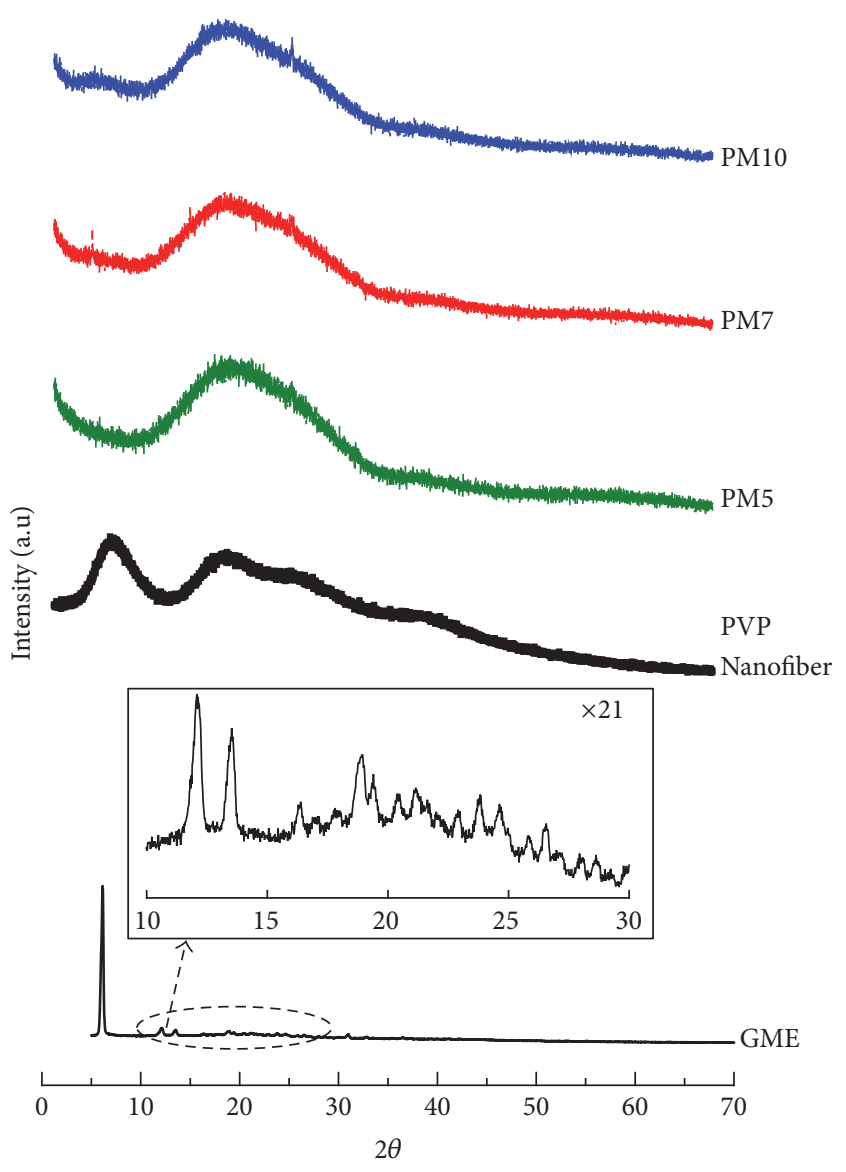

FIGURE 6: XRD patterns of the GME, PVP nanofiber mat, and the PM5, PM7, and PM10 composite nanofiber mats.

reorientation and thus the molecules were in a lower order. Such transition has also been obtained from the XRD studies of electrospun emodine/PVP nanofibers [34].

The presence of GME was found to alter the diffraction pattern of PVP. All PVP/GME composite nanofiber mats exhibited a single broad halo that resembled diffusion between the second halo and its shoulder over the $2 \theta$ position of $15-35^{\circ}$. This broad halo peaked at the $2 \theta$ positions of $27.52^{\circ}$ (the PM5 composite nanofiber mat), $26.98^{\circ}$ (the PM7 mat), and $25.31^{\circ}$ (the PM10 mat). Meanwhile, the first halo of PVP nanofiber at the $2 \theta$ position of $10.18^{\circ}$ disappeared. This finding strengthened our presumption that GME and PVP formed intermolecular interaction, particularly as hydrogen bonds, as explained in the FTIR studies. The hydroxyl groups in GME are strong hydrogen bond donors and the carbonyl groups in PVP are strong hydrogen bond acceptors. Therefore, a high number of hydrogen bonds can be formed between GME and PVP in the PM5, PM7, and PM10 composite nanofiber mats. Under this condition, GME can be dispersed in the PVP polymer matrix in molecular levels, not as dimers in crystal lattice. This is an advantageous feature of the electrospun PVP nanofibers as a drug delivery system, which can improve the stability of dispersed drug [34].
TABLE 3: Antioxidant activities of PVP/GME composite nanofiber mats and GME.

\begin{tabular}{lcc}
\hline Sample & Average fiber diameter $(\mathrm{nm})$ & $\mathrm{IC}_{50}(\mu \mathrm{g} / \mathrm{mL})$ \\
\hline PM5 nanofiber mat & $217 \pm 82$ & $45.94 \pm 0.32$ \\
PM7 nanofiber mat & $323 \pm 128$ & $47.93 \pm 0.22$ \\
PM10 nanofiber mat & $421 \pm 174$ & $48.41 \pm 0.24$ \\
GME & - & $50.94 \pm 0.71$ \\
\hline
\end{tabular}

3.5. Antioxidant Activities. A high number of studies has reported the abundant amount of xanthones in Garcinia mangostana L. extract [35]. Xanthones have demonstrated antioxidant activity owing to excellent hydrogen- and electron-donor ability. In our study, DPPH acted as free radical, which is capable of accepting an electron donor from an antioxidant compound to revert eventually to a stable/nonradical molecule. The product of this reduction was indicated by the change in color from violet to pale yellow [1]. Antioxidant activity in this study was described by an $\mathrm{IC}_{50}$ value, in which a stronger antioxidant activity is indicated by a lower $\mathrm{IC}_{50}$ value $[36,37]$. A compound is stated to be "very high" if the $\mathrm{IC}_{50}$ value is within the range of 1 to $50 \mu \mathrm{g} / \mathrm{mL}$, "high" if the value is within 50 to $100 \mu \mathrm{g} / \mathrm{mL}$, "moderate" if the value is within 101 to $150 \mu \mathrm{g} / \mathrm{mL}$, and "weak" if the value is larger than $150 \mu \mathrm{g} / \mathrm{mL}$ [38].

Table 3 compares the antioxidant activity of the GME and the PVP/GME composite nanofiber mats. It was found that the PM5, PM7, and PM10 composite nanofiber mats exhibited very high antioxidant activity larger than that of GME. These findings showed that the antioxidant activity of PM5, PM7, and PM10 composite nanofibers was higher than the GME although it had been exposed to high voltages during the electrospinning process. This result corroborated that the previous study that the antioxidant activity of GME was not affected by the electrospinning process [39].

As previously mentioned, nanofibers with the smallest diameter exhibited the strongest antioxidant activity. It was then suggested that the antioxidant activity of GME was enhanced due to nanostructure offered by the nanofiber mats. Small-diameter fibers possess higher surface area [40] which, in turn, means that more $\alpha$-mangostin molecules are available on the surface of the fiber. As a result, $\alpha$-mangostin can demonstrate antioxidant activity more readily [21]. Previous study has reported similar finding where antioxidant activity of polyaniline (PANI) increased after being electrospun into nanofiber form [41]. This study also showed that antioxidant activity of PANI was enhanced by decreasing fiber diameter. Therefore, the PVP/GME composite nanofibers are potential as antioxidant products to be applied in food and pharmaceutical industries.

\section{Conclusion}

We have successfully prepared PVP/GME composite nanofiber mats using electrospinning method. The smooth morphology and nanosized diameter of the composite fibers as given by their SEM images indicated sufficient polymer concentration, good spinnability, and high surface area. The 
latter is advantageous in drug delivery systems for poorly water-soluble drugs. A high number of hydrogen bonds and a single broad halo of the composite nanofiber mats as confirmed by their FTIR spectra and XRD patterns, respectively, suggested molecularly dispersed GME in the electrospun PVP polymer nanofibers. This is a beneficial feature of the electrospun PVP nanofibers as a drug delivery system for improving the stability of dispersed drug such as GME. Moreover, the composite nanofiber mats showed very strong antioxidant activities, indicating that high voltage exposure during the electrospinning process did not affect the antioxidant activities. These findings suggested that the composite nanofiber mat could find application in food and pharmaceutical industries as a potential antioxidant product.

\section{Conflicts of Interest}

The authors declare that they have no conflicts of interest.

\section{Acknowledgments}

This research was financially supported by Directorate of Research and Community Engagement of Ministry of Research, Technology and Higher Education, the Republic of Indonesia, under the University's Excellence Research (PUPT) Grant in the fiscal year 2015-2016.

\section{References}

[1] J. Pedraza-Chaverri, N. Cárdenas-Rodríguez, M. OrozcoIbarra, and J. M. Pérez-Rojas, "Medicinal properties of mangosteen (Garcinia mangostana)," Food and Chemical Toxicology, vol. 46, no. 10, pp. 3227-3239, 2008.

[2] Y. M. Chong, S. K. Chang, W. C. M. Sia, and H. S. Yim, "Antioxidant efficacy of mangosteen (Garcinia mangostana Linn.) peel extracts in sunflower oil during accelerated storage," Food Bioscience, vol. 12, article 112, pp. 18-25, 2015.

[3] M. Naczk, M. Towsend, R. Zadernowski, and F. Shahidi, "Protein-binding and antioxidant potential of phenolics of mangosteen fruit (Garcinia mangostana)," Food Chemistry, vol. 128, no. 2, pp. 292-298, 2011.

[4] H.-A. Jung, B.-N. Su, W. J. Keller, R. G. Mehta, and A. D. Kinghorn, "Antioxidant xanthones from the pericarp of Garcinia mangostana (Mangosteen)," Journal of Agricultural and Food Chemistry, vol. 54, no. 6, pp. 2077-2082, 2006.

[5] M. Ahmad, B. M. Yamin, and A. Mat Lazim, "A study on dispersion and characterisation of $\alpha$-mangostin loaded $\mathrm{pH}$ sensitive microgel systems," Chemistry Central Journal, vol. 7, no. 1, p. $85,2013$.

[6] A. F. A. Aisha, Z. Ismail, K. M. Abu-salah, and A. M. S. A. Majid, "Solid dispersions of $\alpha$-mangostin improve its aqueous solubility through self-assembly of nanomicelles," Journal of Pharmaceutical Sciences, vol. 101, no. 2, pp. 815-825, 2012.

[7] A. A. E. Ali, M. Taher, and F. Mohamed, "Microencapsulation of alpha-mangostin into PLGA microspheres and optimization using response surface methodology intended for pulmonary delivery," Journal of Microencapsulation, vol. 30, no. 8, pp. 728740, 2013.

[8] W. Samprasit, R. Kaomongkolgit, M. Sukma, T. Rojanarata, T. Ngawhirunpat, and P. Opanasopit, "Mucoadhesive electrospun chitosan-based nanofibre mats for dental caries prevention," Carbohydrate Polymers, vol. 117, pp. 933-940, 2015.

[9] S. Ramakrishna, K. Fujihara, W. Teo, T. Lim, and Z. Ma, An Introduction to Electrospinning and Nanofibers, World Scientific, Singapore, 2005.

[10] C. Risdian, M. Nasir, A. Rahma, and H. Rachmawati, "The influence of formula and process on physical properties and the release profile of PVA/BSA nanofibers formed by electrospinning technique," Journal of Nano Research, vol. 31, pp. 103-116, 2015.

[11] M. M. Munir, F. Iskandar, Khairurrijal, and K. Okuyama, "High performance electrospinning system for fabricating highly uniform polymer nanofibers," Review of Scientific Instruments, vol. 80, no. 2, Article ID 026106, 2009.

[12] G. Yang, J. Li, D. Yu, M. He, J. Yang, and G. R. Williams, "Nanosized sustained-release drug depots fabricated using modified tri-axial electrospinning," Acta Biomaterialia, vol. 53, pp. 233-241, 2017.

[13] D. Yu, J. Li, M. Zhang, and G. R. Williams, "High-quality Janus nanofibers prepared using three-fluid electrospinning," Chemical Communications, vol. 53, no. 33, pp. 4542-4545, 2017.

[14] D.-G. Yu, X.-Y. Li, X. Wang, J.-H. Yang, S. W. Annie Bligh, and G. R. Williams, "Nanofibers fabricated using triaxial electrospinning as zero order drug delivery systems," ACS Applied Materials \& Interfaces, vol. 7, no. 33, pp. 18891-18897, 2015.

[15] J. Y. Park, I. H. Lee, and G. N. Bea, "Optimization of the electrospinning conditions for preparation of nanofibers from polyvinylacetate (PVAc) in ethanol solvent," Journal of Industrial and Engineering Chemistry, vol. 14, no. 6, pp. 707-713, 2008.

[16] D. G. Yu, X. Wang, X. Y. Li, W. Chian, Y. Li, and Y. Z. Liao, "Electrospun biphasic drug release polyvinylpyrrolidone/ethyl cellulose core/sheath nanofibers," Acta Biomaterialia, vol. 9, no. 3, pp. 5665-5672, 2013.

[17] Y.-N. Jiang, H.-Y. Mo, and D.-G. Yu, "Electrospun drug-loaded core-sheath PVP/zein nanofibers for biphasic drug release," International Journal of Pharmaceutics, vol. 438, no. 1-2, pp. 232239, 2012.

[18] I. Sriyanti, D. Edikresnha, M. M. Munir, H. Rachmawati et al., "Electrospun Polyvinylpyrrolidone (PVP) Nanofiber Mats Loaded by Garcinia mangostana L. extracts," Materials Science Forum, vol. 880, pp. 11-14, 2016.

[19] X.-Y. Li, X. Wang, D.-G. Yu et al., "Electrospun borneol-PVP nanocomposites," Journal of Nanomaterials, vol. 2012, Article ID 731382, 8 pages, 2012.

[20] A. Rahma, M. M. Munir, Khairurrijal, A. Prasetyo, V. Suendo, and H. Rachmawati, "Intermolecular Interactions and the Release Pattern of Electrospun CurcuminPolyvinyl(pyrrolidone) Fiber," Biological and Pharmaceutical Bulletin, vol. 39, no. 2, pp. 163-173, 2016.

[21] C. Palakawong, P. Sophanodora, S. Pisuchpen, and S. Phongpaichit, "Antioxidant and antimicrobial activities of crude extracts from mangosteen (Garcinia mangostana L.) parts and some essential oils," International Food Research Journal, vol. 17, no. 3, pp. 583-589, 2010.

[22] P. P. Bhat, S. Appathurai, M. T. Harris, M. Pasquali, G. H. McKinley, and O. A. Basaran, "Formation of beads-on-a-string structures during break-up of viscoelastic filaments," Nature Physics, vol. 6, no. 8, pp. 625-631, 2010.

[23] H. Fong, I. Chun, and D. H. Reneker, "Beaded nanofibers formed during electrospinning," Polymer, vol. 40, no. 16, pp. 4585-4592, 1999. 
[24] C. Clasen, J. Bico, V. M. Entov, and G. H. McKinley, “Gobbling drops': the jetting-dripping transition in flows of polymer solutions," Journal of Fluid Mechanics, vol. 636, pp. 5-40, 2009.

[25] T. Vongsetskul, T. Chantarodsakun, P. Wongsomboon, R. Rangkupan, and P. Tangboriboonrat, "Effect of solvent and processing parameters on electrospun polyvinylpyrrolidone ultra-fine fibers," Chiang Mai Journal of Science, vol. 42, no. 2, pp. 436-442, 2015.

[26] J. Matulevicius, L. Kliucininkas, T. Prasauskas, D. Buivydiene, and D. Martuzevicius, "The comparative study of aerosol filtration by electrospun polyamide, polyvinyl acetate, polyacrylonitrile and cellulose acetate nanofiber media," Journal of Aerosol Science, vol. 92, pp. 27-37, 2016.

[27] M. Guo, X. Wang, X. Lu, H. Wang, and P. E. Brodelius, " $\alpha$ Mangostin extraction from the native mangosteen (Garcinia mangostana $\mathrm{L}$ ) and the binding mechanisms of $\alpha$-mangostin to HSA or TRF," PLoS ONE, vol. 11, no. 9, Article ID e0161566, 2016.

[28] S. A. Sheikh Mohd Ghazali, G. Ee Cheng Lian, and K. D. Abd Ghani, "Chemical Constituent from Roots of Garcinia Mangostana (Linn.)," International Journal of Chemistry, vol. 2, no. 1, pp. 134-142, 2010.

[29] C. Jing and J. Hou, "Sol-gel-derived alumina/polyvinylpyrrolidone hybrid nanocomposite film on metal for corrosion resistance," Journal of Applied Polymer Science, vol. 105, no. 2, pp. 697-705, 2007.

[30] A. L. Saroj, R. K. Singh, and S. Chandra, "Studies on polymer electrolyte poly(vinyl) pyrrolidone (PVP) complexed with ionic liquid: Effect of complexation on thermal stability, conductivity and relaxation behaviour," Materials Science and Engineering B: Solid-State Materials for Advanced Technology, vol. 178, no. 4, pp. 231-238, 2013.

[31] C. M. Laot, E. Marand, and H. T. Oyama, "Spectroscopic characterization of molecular interdiffusion at a poly(vinyl pyrrolidone)/vinyl ester interface," Polymer, vol. 40, no. 5, pp. 1095-1108, 1999.

[32] J. P. M. Lommerse, S. L. Price, and R. Taylor, "Hydrogen bonding of carbonyl, ether, and ester oxygen atoms with alkanol hydroxyl groups," Journal of Computational Chemistry, vol. 18, no. 6, pp. 757-774, 1997.

[33] W. Samprasit, T. Rojanarata, P. Akkaramongkolporn, T. Ngawhirunpat, R. Kaomongkolgit, and P. Opanasopit, "Fabrication and in vitro/in vivo performance of mucoadhesive electrospun nanofiber mats containing $\alpha$-mangostin," AAPS PharmSciTech, vol. 16, no. 5, pp. 1140-1152, 2015.

[34] X.-Y. Dai, W. Nie, Y.-C. Wang, Y. Shen, Y. Li, and S.-J. Gan, "Electrospun emodin polyvinylpyrrolidone blended nanofibrous membrane: a novel medicated biomaterial for drug delivery and accelerated wound healing," Journal of Materials Science: Materials in Medicine, vol. 23, no. 11, pp. 2709-2716, 2012.

[35] Y.-L. Huang, C.-C. Chen, Y.-J. Chen, R.-L. Huang, and B.-J. Shieh, "Three xanthones and a benzophenone from Garcinia mangostana," Journal of Natural Products, vol. 64, no. 7, pp. 903906, 2001.

[36] M. S. Blois, "Antioxidant determinations by the use of a stable free radical," Nature, vol. 181, no. 4617, pp. 1199-1200, 1958.

[37] P. Molyneux, "The use of the stable free radical diphenylpicrylhydrazyl (DPPH) for estimating antioxidant activity," Songklanakarin Journal of Science and Technology, vol. 26, no. 2, pp. 211-219, 2004.

[38] I. Fidrianny, S. Natalia, and M. Insanu, "Antioxidant capacities of various fruit extracts from three varieties of tomato and correlation with total phenolic, flavonoid, carotenoid content," International Journal of Pharmaceutical and Clinical Research, vol. 7, no. 4, pp. 283-289, 2015.

[39] N. Charernsriwilaiwat, T. Rojanarata, T. Ngawhirunpat, M. Sukma, and P. Opanasopit, "Electrospun chitosan-based nanofiber mats loaded with Garcinia mangostana extracts," International Journal of Pharmaceutics, vol. 452, no. 1-2, pp. 333-343, 2013.

[40] Z.-M. Huang, Y. Z. Zhang, M. Kotaki, and S. Ramakrishna, "A review on polymer nanofibers by electrospinning and their applications in nanocomposites," Composites Science and Technology, vol. 63, no. 15, pp. 2223-2253, 2003.

[41] J. Wang, L. H. Zhu, J. Li, and H. Q. Tang, "Antioxidant activity of polyaniline nanofibers," Chinese Chemical Letters, vol. 18, no. 8, pp. 1005-1008, 2007. 

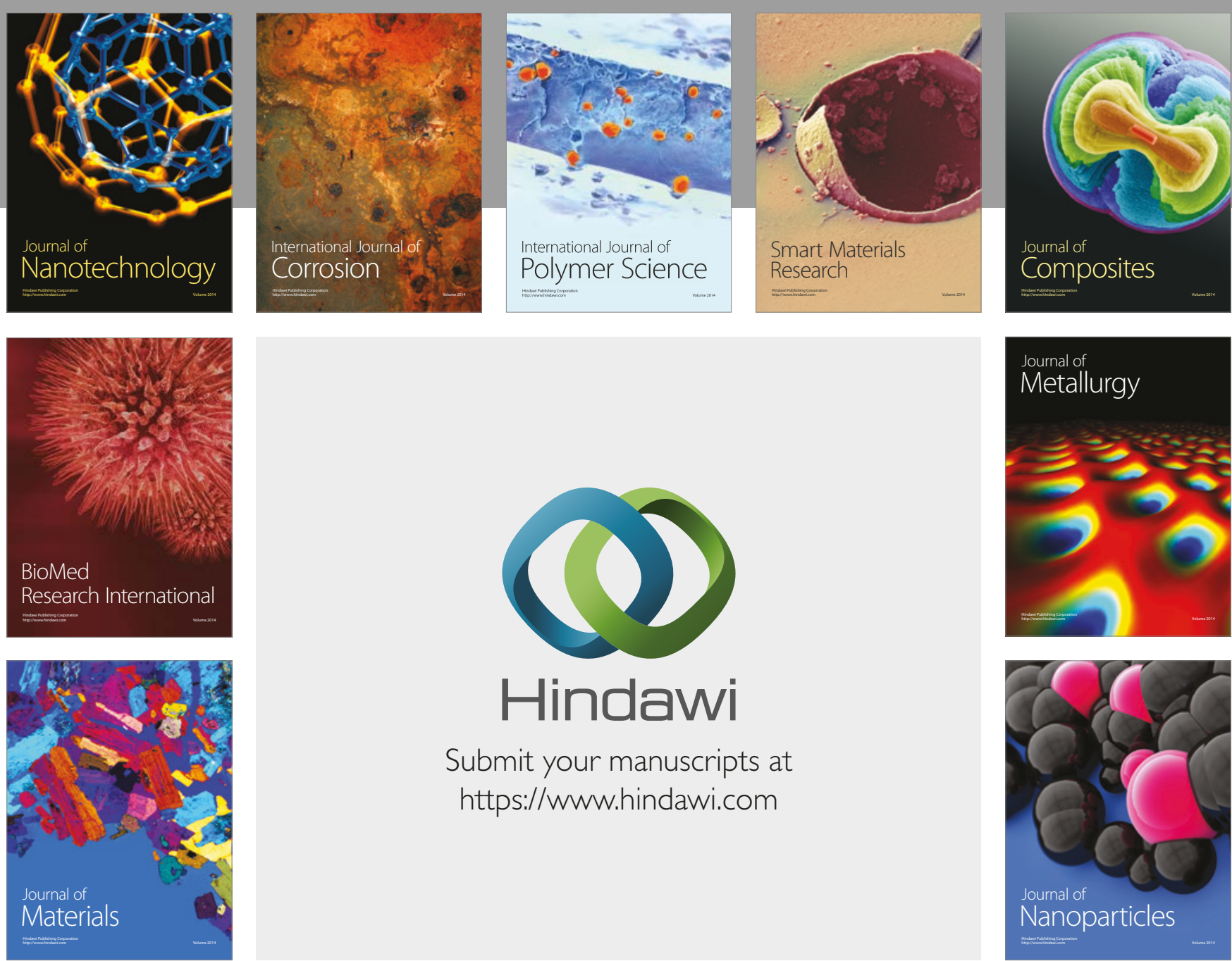

\section{Hindawi}

Submit your manuscripts at

https://www.hindawi.com
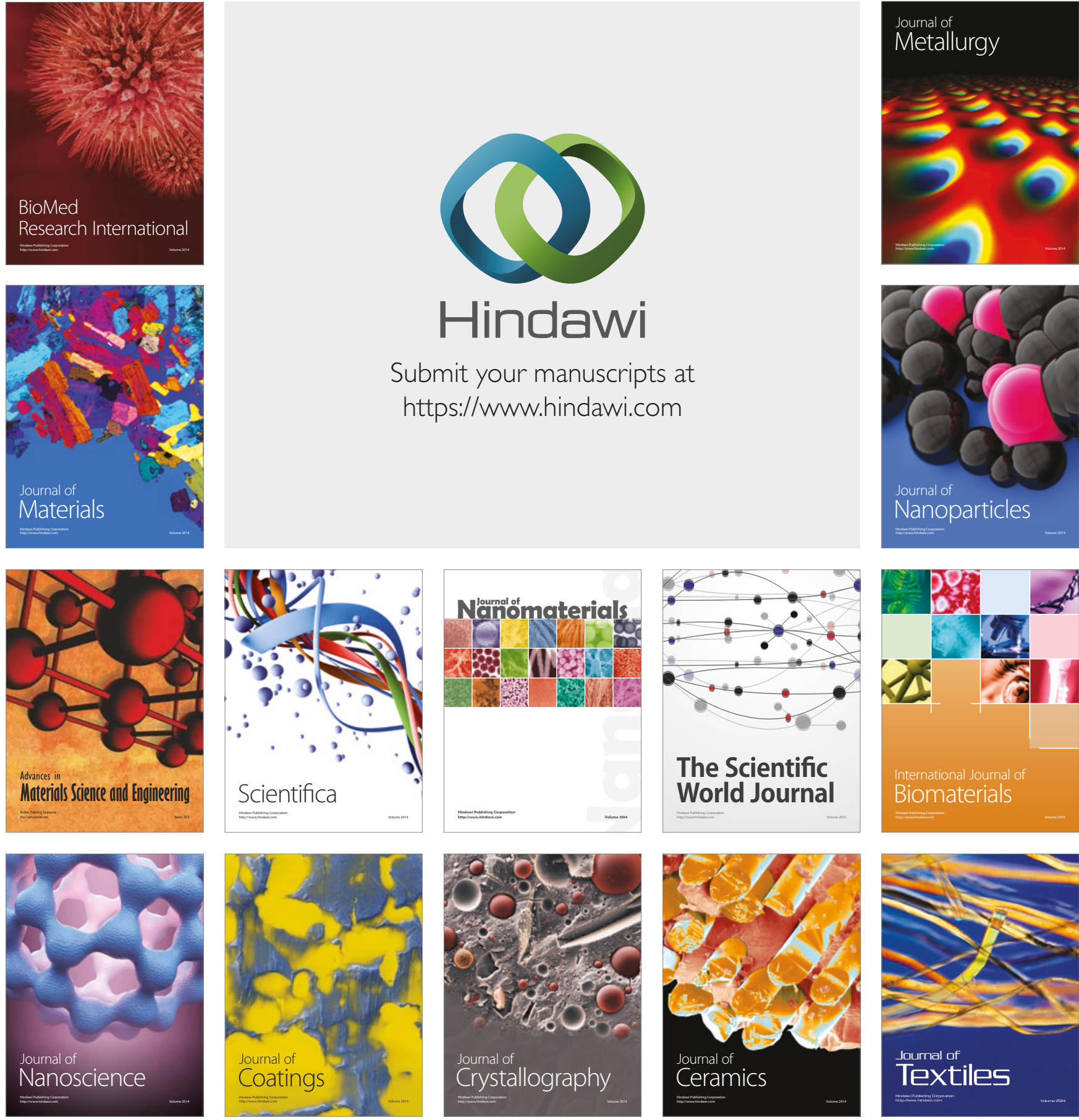

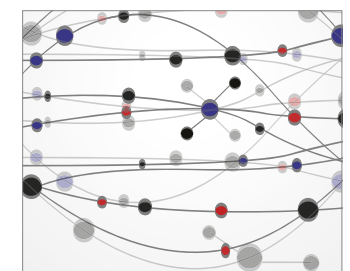

The Scientific World Journal
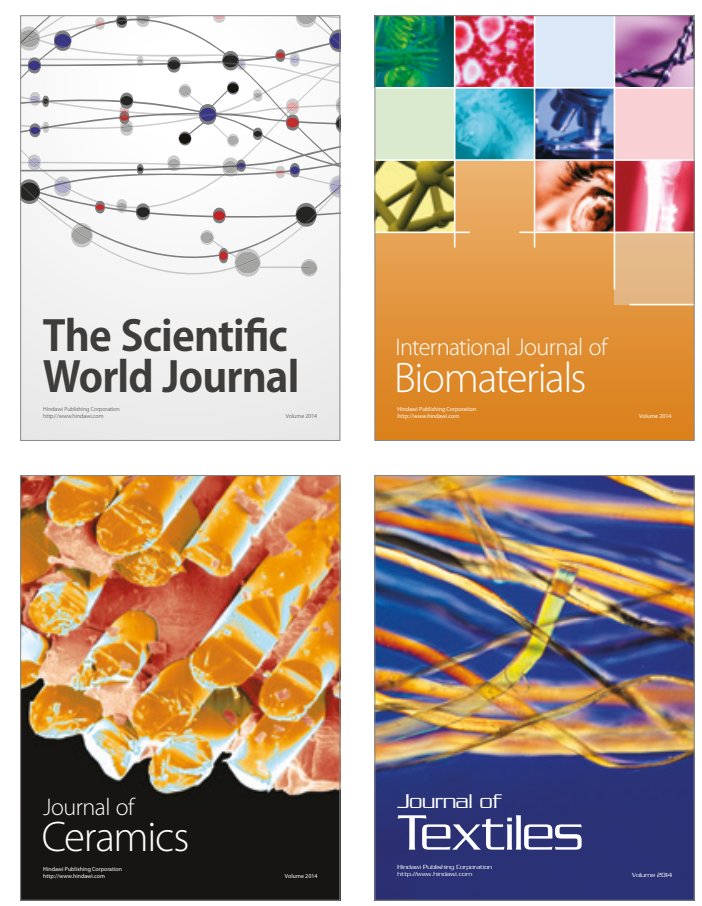